\title{
Implicaciones académicas del conocimiento metacognitivo: estudio de caso con estudiantes de Diplomado en Enseñanza del Inglés
}

\author{
Academic implications of metacognitive knowledge: \\ case study with first-year students of the English Teaching Major
}

\author{
Emilia María Quirós Vargas ${ }^{1}$ \\ Universidad Estatal a Distancia, Costa Rica
}

\begin{abstract}
Resumen. La UNED es la única universidad costarricense que ofrece la Carrera de Enseñanza del Inglés a través de la modalidad a distancia. El artículo presenta los aspectos más destacados de un estudio sobre la naturaleza del conocimiento metacognitivo (CMC) de estudiantes de primer año y las implicaciones en su rendimiento académico. Siete estudiantes de la cohorte de 2017 participaron en un estudio de caso múltiple para evaluar su CMC basándose en cuatro categorías de conocimiento descritas en el modelo de Flavell-Pintrich: persona, tarea, estrategia y contexto. Además, de una entrevista semiestructurada y dos encuestas en línea para recolección de datos, se analizó el desempeño de los participantes durante el cuatrimestre, sus calificaciones, las características de su contexto instruccional y la evaluación cualitativa desde la perspectiva de sus tutores. Los resultados indicaron el valor del conocimiento de la persona en el proceso formativo. Este constituye el filtro de la información que llega al individuo y, por lo tanto, determina, en gran medida, su percepción del tipo de tarea que se le presenta, así como las estrategias que se utilizarán para su resolución. Se concluye que, aunque un estudiante tiene facilidades y acceso para el uso de la tecnología, es el autoconocimiento el que concentra los recursos cognitivos de una manera particular para abordar y resolver problemas de aprendizaje.
\end{abstract}

Palabras clave. Metacognición, conocimiento metacognitivo, aprendizaje de idiomas a distancia

\begin{abstract}
WUNED is the only Costa Rican university that offers the English teaching major through the distance learning modality. The article presents highlights of a study on the nature of metacognitive knowledge (MCK) of first-year students and its implications on their academic performance. Seven students from the 2017 cohort participated in a multiple case study to evaluate their CMC based on four dimensions of knowledge described in the Flavell-Pintrich model: person, task, strategy and context. In addition to a semi-structured interview and two online surveys for data collection, the participants' performance in the academic term, their grades, the elements of their instructional context, and qualitative evaluations from their tutors were thoroughly analyzed. The results highlighted the value of the person's knowledge of the person in the training process. This knowledge constitutes the filter of the information that reaches the individual and, therefore, largely determines his or her of the type of task that is presented, as well as the strategies that will be used to solve it. It can be assumed that, although a student has knowledge and access to the use of technology, it is personal knowledge that concentrates cognitive resources in a particular way to address and solve learning problems.
\end{abstract}

Keywords. Metacognition, metacognitive knowledge, language distance learning

${ }^{1}$ Doctora en Educación. Es coordinadora de las cátedras de Inglés Técnico y de Lengua y Cultura Inglesa en la Carrera de Enseñanza del Inglés para I y II ciclos en la Escuela de Ciencias Sociales, Universidad Estatal a Distancia, Costa Rica. Dirección electrónica: equiros@uned.ac.cr 


\section{Introducción}

El papel de la metacognición es clave en contextos de aprendizaje a distancia. Debido a la separación de docentes y estudiantes en espacio y tiempo, se hace uso intensivo de herramientas tecnológicas en momentos y lugares que los educandos deben determinar. El estudiantado en esta modalidad debe utilizar una variedad de tecnologías, medios y recursos para lograr la integración de los componentes de aprendizaje $\mathrm{y}$ establecer interacciones efectivas (Hong y Jung, 2011). Sin embargo, se ha encontrado que no siempre está preparado para emplear, de manera eficaz, las herramientas ni los recursos en entornos mediados por la tecnología, así como tampoco para maximizar su experiencia educativa y lograr mejores resultados (Gurbin, 2015).

Diversos estudios muestran que el aprendizaje a distancia demanda mayor uso de estrategias metacognitivas que la modalidad presencial o tradicional (Hampel y de los Arcos, 2013). Factores contextuales tales como las actividades de aprendizaje y los materiales, el diseño de tareas, los modos de interacción y la formación de los y las estudiantes pueden contribuir a su desarrollo metacognitivo $y$, por ende, lograr una mejor auto-regulación (White, 2014).

En el caso particular de la UNED, el número de estudiantes que ingresan para formarse como docentes de inglés aumenta cada año, pero los estudios sobre el conocimiento metacognitivo (CMC) que posee el alumnado en entornos de aprendizaje no tradicionales y las repercusiones que este tiene en su logro académico, son más bien escasos. De tal manera la investigación sobre los factores contextuales de la educación a distancia y su relación con el desempeño observable de los y las estudiantes de inglés son necesarios para comprender mejor cómo puede propiciarse una enseñanza efectiva a través de las características del diseño instruccional y qué tipo de intervenciones se requieren para fortalecer la autorregulación y la autonomía mediante el desarrollo metacognitivo.

Existen varios factores que añaden complejidad al proceso educativo de los y las estudiantes de enseñanza del inglés de la UNED. Uno de ellos es que el alumnado debe adaptarse a una modalidad mediada por tecnología, transición ya de por sí difícil, en especial en las fases iniciales en la universidad. El estudio a distancia también demanda habilidades particulares para la planificación de tareas y administración del tiempo, a fin de cumplir con plazos de entrega ajustados a la calendarización cuatrimestral.

Otro factor reside en el hecho de que todas las asignaturas de formación docente son impartidas en inglés. Es decir, este idioma no solo es objeto de estudio, sino también es el medio de comunicación e intercambio de información. Considerando lo anterior, es comprensible que estudiantes que carecen de estrategias eficaces y actitudes adecuadas, motivación y capacidad para regular o auto controlar su aprendizaje, deserten del sistema o inviertan más tiempo en la obtención del título universitario.

En relación con el plano nacional, los nuevos programas del Ministerio de Educación Pública para la enseñanza del inglés en escuelas y colegios de Costa Rica se concentran en desarrollar competencias lingüísticas mediante una formación integral para que los y las aprendices sean capaces de comunicarse en el idioma meta, por ejemplo, a través de discusiones y debates en clase. Estos programas se apoyan en una mediación docente efectiva, lo que destaca la responsabilidad que tienen las universidades de preparar a los futuros educadores y educadoras en capacidades de pensamiento, comunicación, toma de decisiones, observación, elaboración de propuestas, creatividad, experimentación, búsqueda $\mathrm{y}$ selección del conocimiento que requieran. 
Tomando en consideración que la Carrera de Enseñanza del inglés de la UNED se enfoca en la formación de docentes de inglés para primaria, es de suma importancia incentivar en el estudiantado el desarrollo de competencias no solo lingüísticas sino cognitivas y metacognitivas que puedan potenciarse con el tiempo. Esto puede lograrse a través de un plan de estudio que promueva el perfeccionamiento lingüístico y pedagógico, así como el desarrollo de conocimientos y destrezas metacognitivas que puedan utilizarse con eficiencia en diversos ámbitos educativos. De aquí parte la necesidad de desarrollar la presente investigación.

Como objetivo general, se propuso evaluar el CMC de estudiantes del Diplomado de la Carrera de Enseñanza del Inglés para I y II ciclo de la UNED que participaron en el estudio. Sobre esta línea, los objetivos específicos del estudio apuntaron a la caracterización del CMC de los participantes, según las categorías de conocimiento de la persona, de la tarea, de las estrategias y del contexto de aprendizaje, con base en el modelo de Flavell-Pintrich que se detallará en el próximo apartado. Por último, la investigación se propuso integrar los hallazgos sobre los conocimientos de los y las participantes para determinar su incidencia en el desempeño académico de los mismos.

La metodología propuesta para la presente investigación fue seleccionada por su correspondencia con la naturaleza del tema bajo escrutinio. Los estudios de caso se enfocan en un solo individuo o pequeño grupo de personas, lo que permite un examen cercano y profundo, así como la recopilación de una gran cantidad de datos detallados. Por otra parte, permiten el uso de varias técnicas distintas para obtener la información necesaria. Si bien los estudios de caso no prueban hipótesis, sugieren direcciones para estudios subsecuentes, lo que se considera un valor agregado de la investigación.

\section{Fundamentación teórica}

En un principio, la metacognición fue definida como el conocimiento y regulación de las actividades cognoscitivas (Flavell, 1979). La investigación en diferentes disciplinas ha indicado que lo que distingue a estudiantes más exitosos es su capacidad de controlar de manera consciente su proceso de aprendizaje y pensamiento, es decir su metacognición (Kempe, 2011).

John Flavell, psicólogo estadounidense, acuñó el término metacognición en la década de 1970 en el sentido de "cognición acerca de los fenómenos cognitivos", en otras palabras, "pensar sobre el pensamiento" (Flavell, 1979). La metacognición involucra el conocimiento de tres variables, a saber, persona, tarea y estrategia. El estudio que da pie a este artículo incorpora una categoría adicional sugerida por otro psicólogo estadounidense, Paul Pintrich: el conocimiento contextual y condicional (Pintrich, 2002). A continuación, se presenta una breve descripción de cada una de las categorías que constituyen el conocimiento metacognitivo (CMC) de acuerdo con el modelo Flavell-Pintrich.

\section{Conocimiento de la persona}

Esta categoría se refiere a las creencias que los individuos poseen acerca de sí mismo como aprendiz (variables intraindividuales), y lo que cree acerca de los procesos de pensamiento de otras personas (variables interindividuales). Las creencias acerca de uno mismo (autoconcepto) pueden facilitar o impedir un buen desempeño en situaciones de aprendizaje (Flavell, 1987).

En el aprendizaje de una segunda lengua, el conocimiento de la persona se refiere a factores cognitivos tales como las evaluaciones que los estudiantes hacen de su propio dominio del idioma meta, o bien, sus fortalezas y debilidades en una destreza lingüística, específica, los problemas que pueden tener y su rendimiento 
en una determinada tarea. Un estudiante tiene creencias motivacionales que se expresan en la eficacia auto-percibida, las metas propuestas para completar una tarea, el nivel de interés y el valor que una tarea tiene para sí (Flavell, 1987).

\section{Conocimiento de la tarea}

Esta categoría se refiere a la información sobre una tarea que está disponible para la persona que aprende (Flavell, 1979). Es el conocimiento acerca de la naturaleza de una empresa cognitiva y las implicaciones que tiene para la mejor gestión de esta actividad, así como la manera probable de lograr objetivos en forma exitosa (Flavell, 1979). Tiene tres facetas que abarcan la comprensión de una tarea cognitiva en términos de objetivo, propósito y demandas sobre la base de conocimientos y habilidades (Cohen, 2011). Estos datos le guían en la gestión de un trabajo determinado y le proporcionan orientación sobre el grado de éxito probable.

La primera faceta se refiere a lo que los estudiantes conocen acerca del propósito de una tarea y cómo servirá esta a sus necesidades de aprendizaje; por ejemplo, si le ayudará a mejorar sus habilidades de escritura, ampliar su vocabulario o desarrollar fluidez en la comunicación oral. La segunda faceta atañe al proceso de clasificación para establecer la naturaleza de una tarea en particular. Por ejemplo, el estudiante concluye que aprender a leer no es lo mismo que aprender a escribir, o que una asignación de pensamiento creativo no es igual a una tarea de resolución de problemas.

Por último, el conocimiento de la tarea aporta información sobre sus demandas, procedimientos y las competencias que se necesitan para ejecutarla. Por ejemplo, la persona puede ser consciente de que escribir un ensayo persuasivo, informativo o descriptivo requiere diferentes tipos de habilidades de composición según sea el caso (Cohen, 2011).

\section{Conocimiento de la estrategia}

Este conocimiento se refiere a la comprensión de cuáles estrategias suelen ser eficaces en el logro de metas particulares, según el tipo de compromisos cognitivos (Flavell, 1979). El mismo abarca la identificación de objetivos y sub-objetivos en una determinada tarea, asimismo los procedimientos cognitivos que deben utilizarse para su logro.

Las acciones que pueden emplearse son cognitivas y metacognitivas. Las cognitivas son esencialmente tres: ensayo, elaboración y organización. Las estrategias de ensayo se caracterizan por la memorización de palabras y no se consideran eficaces para procesos cognitivos más complejos. La elaboración (resumir, parafrasear, identificar la idea central) y la organización (elaborar esquemas, mapas conceptuales y tomar notas), por lo general, dan como resultado mejor comprensión y aprendizaje (Raoofi et al, 2013).

Además de este tipo de procedimientos, existen otros que resultan esenciales para el aprendizaje: el planeamiento, el monitoreo, y la auto-regulación del aprendizaje y el pensamiento (Flavell, 1979, 1987). La planificación implica el establecimiento de metas, el análisis de tareas, la selección de técnicas apropiadas y la asignación de recursos que afectan el desempeño.

El monitoreo incluye procedimientos de comprobación como el auto cuestionamiento y el autocontrol. La evaluación se refiere a la valoración de los productos y la eficiencia de lo que se ha aprendido. Ejemplos de ello son la evaluación de las estrategias empleadas y la reevaluación de los objetivos.

\section{Conocimiento del contexto de aprendizaje}

El conocimiento contextual y condicional es básicamente la conciencia que una persona tiene acerca de las condiciones de su entorno que son propicias para la realización de tareas específicas. 
Implica comprensión de las normas locales, generales y situacionales, convencionales, sociales y culturales que, en apariencia, afectan la cognición (Pintrich et al., 2002; Richards y Rodgers, 2014).

La conciencia del contexto informa a los o las aprendices acerca de cómo, cuándo y por qué deben utilizar ciertas estrategias (Pintrich et al., 2002). Integra las normas locales, generales y situacionales, convencionales, sociales y culturales que, en mayor o menor medida, afectan la cognición del alumno (Pintrich et al., 2002; Richards y Rodgers, 2014). En el caso de la modalidad a distancia, el contexto instruccional es determinante, en especial, si se involucra el componente tecnológico. La ansiedad generada por el uso de la computadora, manifestada en nerviosismo y malestar generalizado al interactuar con herramientas o aplicaciones virtuales, constituye un elemento crítico en la predicción de logro en el aprendizaje en línea.

El conocimiento metacognitivo o CMC está relacionado con la transferencia del aprendizaje (Dewitz y Graves, 2014). Cuando las personas se enfrentan a nuevas tareas o s situaciones donde sus habilidades y nociones específicas del dominio son insuficientes, sus conocimientos de estrategias de aprendizaje generales pueden facilitarel abordaje de tareas nuevas o desafiantes. El CMC también viene a ser un requisito previo a la autorregulación, permitiendo a los y las estudiantes ser agentes activos de su propio aprendizaje. El autoconocimiento puede influir además en la elección de objetivos y metas, los cuales actuarán entonces como los criterios a utilizar para la evaluación y supervisión del proceso. De igual modo, el CMC facilita la identificación de acciones cognitivas con las que se pueden alcanzar metas y maximizar el aprovechamiento académico.

Aunque el CMC no necesita ser articulado en forma explícita para que pueda ser utilizado, se ha argumentado que una mayor conciencia metacognitiva, puede facilitar considerablemente el pensamiento y la autorregulación (Kormos y Cscizer, 2014). De hecho, se ha comprobado que la capacidad que un estudiante posee para comunicar y discutir de manera verbal sus ideas sobre cognición, por ejemplo, la forma en que aborda una asignación, indica su nivel de comprensión sobre el tema.

\section{Metodología}

Mediante un estudio de caso múltiple desarrollado por la autora de esta ponencia para su tesis doctoral en el año 2017 con estudiantes de primer ingreso de la Carrera de Enseñanza del inglés de la UNED, fue posible identificar puntos de coincidencia con la base teórica generada por investigaciones precedentes relativas al CMC y su incidencia en el desempeño académico de los alumnos.

El paradigma que se adoptó en este estudio fue el interpretativo (Hernández, Fernández y Baptista, 2010). El mismo intenta sustituir las nociones científicas de explicación, predicción y control, propios del positivismo, por las de comprensión, significado y acción. Se centra en el estudio de los significados de las acciones humanas y de la vida social y busca la objetividad en el ámbito de los significados pues utiliza como criterio de evidencia el acuerdo intersubjetivo en el contexto educativo. Desde esta perspectiva se cuestiona que el comportamiento de los sujetos este gobernado por leyes generales y caracterizado por regularidades subyacentes. Los investigadores de orientación interpretativa se centran en la descripción y comprensión de lo que es único y particular del sujeto más que en lo generalizable.

En la investigación cualitativa, el contexto no es controlado o manipulado por el investigador. El esfuerzo por comprender la perspectiva de los alumnos y alumnas requiere el uso de técnicas, 
como las entrevistas y la observación, para interactuar de manera profunda y cercana con ellos durante el estudio (Gay et. al, 2013). Por esta razón, el número de participantes tiende a ser pequeño y el análisis de datos se hace de manera inductiva mediante la categorización y la organización de patrones o diferencias (o la ausencia misma de hallazgos esperados según la teoría) que producen una síntesis o narración descriptiva (Creswell, 2013).

El estudio de casos, seleccionado como diseño para esta investigación, constituye un medio para el análisis de la realidad social en el desarrollo de las ciencias sociales y humanas y representa la forma más pertinente de las investigaciones orientadas desde una perspectiva cualitativa. Este diseño presenta un perfil humano contextualizado y propicia la exploración a profundidad de un problema o situación particular. En el ámbito del aprendizaje de idiomas, por ejemplo, se han generado nuevas perspectivas y depurado teorías anteriores en lingüística aplicada como resultado del análisis de fenómenos lingüísticos, culturales y sociales asociados con niños y niñas, adolescentes, personas jóvenes y adultas mayores (Duff, 2012).

Los y las participantes del estudio se seleccionaron de acuerdo con la estrategia de muestreo intencional (Creswell, 2013). Para asegurar la diversidad de casos, se siguieron criterios tales como: distintos niveles de competencia en el inglés, estudiantes femeninos y masculinos de diferentes grupos etarios, estado civil, lugar de procedencia y condiciones socioeconómicas diferentes.

Con respecto al número de participantes, las investigaciones en aprendizaje de idiomas coinciden en queunnúmeroidealdeparticipantes en estudios múltiples es de cuatro a seis casos, en especial para investigación doctoral, ya que permite la diversidad de formas de presentación de los resultados; a saber: en pares, en estudios individuales, cruzados o según los temas que abarquen los casos, en lugar de analizarlos uno por uno (Duff, 2008; Stake, 2010).

De esta manera, se invitó al estudiantado de nuevo ingreso a participar en el estudio en febrero de 2017. Se les envió una fórmula de Consentimiento Informado que debía devolverse firmada, en caso de existir interés en ser parte del estudio. Una vez hecha la selección preliminar de participantes, se les envió el enlace para completar la encuesta en línea denominada Escala de preparación para el aprendizaje a distancia con apoyo tecnológico. A los siete estudiantes que cumplieron con los criterios estimados, se le invitó nuevamente en marzo de 2017 para coordinar las siguientes actividades de investigación; a saber: la participación en la Encuesta de antecedentes en aprendizaje de idiomas y uso de tecnología, así como una entrevista extensa.

La Escala de Preparación para el aprendizaje en línea (Hung et al., 2010) fue diseñada con el fin de medir el nivel inicial de preparación que el estudiante tiene para el aprendizaje a distancia con apoyo tecnológico. La investigadora tradujo al español esta escala y la Encuesta de Antecedentes. Adicionalmente, adaptó ambos instrumentos al contexto costarricense para usarlos en el estudio.

Para la construcción de las entrevistas, la investigadora empleó tipos de preguntas generadas en estudios previos sobre metacognición (Graham, 2004) y se complementaron con la aplicación de la técnica de tema acoplado, desarrollada por White (1999), la cual ha probado, con el tiempo, ser de efectividad para obtener información densa sobre el CMC de los estudiantes desde una perspectiva diferente. Otro documento utilizado fue la evaluación cualitativa o reflexión del desempeño general del estudiante por parte de los tutores encargados de los cursos. Dicha reflexión proporcionó una perspectiva diferente sobre el proceso de aprendizaje de los 
participantes y la carrera, y aportó elementos a valorar como parte del contexto instruccional.

Los datos que se obtuvieron a partir de la aplicación de las encuestas en línea se usaron para crear una ficha informativa de cada participante. Todas las entrevistas, excepto una, se hicieron de manera presencial y se grabaron en audio. Además de conocer la condición del CMC del discente en relación con el aprendizaje del inglés en el contexto de la educación a distancia, se deseaba corroborar o ampliar los datos que los alumnos habían reportado tanto en el cuestionario en línea como en la encuesta de antecedentes respecto a sus creencias motivacionales y las estrategias empleadas, esto con el objetivo de precisar temas según las categorías del modelo Flavell-Pintrich que el estudio había adoptado.

Para el análisis de los datos cualitativos se siguió el enfoque inductivo con ayuda del software ATLAS. Ti (Hernández et al., 2010). Los datos que se obtuvieron se fueron procesando de manera continua. La información de cada caso individual se comparó y trianguló con la de las encuestas de los otros casos. El proceso siguió tres líneas concurrentes de actividad: documentación, reducción y visualización de datos y elaboración y verificación de conclusiones (Hernández et al., 2010).

Lastranscripciones delasentrevistas sehicieron a mano para lograr un mayor acercamiento con el material recopilado. No se establecieron categorías preliminares, aunque luego se dio lectura general de cada transcripción para iniciar la codificación del texto, de acuerdo con las dimensiones del conocimiento metacognitivo (Flavell, 1979, Pintrich, 2002).

El proceso de análisis continuó con la revisión sistemática y cronológica de los segmentos de texto para buscar factores contextuales relevantes. Luego, se examinaron otras fuentes de datos para identificar las conexiones: contenidos de las orientaciones académicas, modelos de evaluación, rúbricas de calificación, actividades de aprendizaje en los entornos de las asignaturas en la plataforma virtual de la universidad, metas y objetivos de aprendizaje, requerimientos para los alumnos, recomendaciones al estudiantado para lograr un mejor aprovechamiento en el estudio auto-dirigido del inglés y sugerencias acerca de la administración del tiempo por semana, del estudio independiente y de la elaboración de tareas. La realimentación que brindaron los tutores también fue analizada como parte de los elementos contextuales.

\section{Resultados}

Los datos provenientes de la aplicación de las dos encuestas en línea constataron que el acceso y uso de tecnología no constituía una limitante para el buen desempeño de los estudiantes en esta investigación, ya que no solo disponían del equipo básico, sino que además habían tenido amplia exposición a la tecnología e interacción en entornos virtuales. No obstante, algunos participantes indicaron no sentir confianza para brindar aportes en foros académicos o expresar opiniones mediante el texto electrónico. De hecho, algunos manifestaron que no tenían seguridad para usar Internet para buscar o seleccionar información para el aprendizaje en línea, lo que evidenciaba debilidades en su utilización de medios tecnológicos con fines académicos.

Durante la entrevista, todos los participantes pudieron expresar sus creencias y percepciones acerca de diversas facetas de su rol como estudiantes de idioma a distancia. No obstante, se encontró que este conocimiento no siempre era preciso. En consulta con los tutores encargados y contando con muestras de trabajos de los estudiantes y las calificaciones recibidas, se pudo corroborar que existían discrepancias entre lo que los alumnos creían saber acerca de 
sí mismos y la valoración de su desempeño por parte de otras personas.

En relación con las creencias personales, Anita Wenden se refirió a las "representaciones del estudiante" (Wenden, 1994). Este término proviene de la psicología constructivista y apunta al hecho de que el CMC no es una réplica exacta de la experiencia, sino que se codifica en la memoria a largo plazo en la forma en que es percibida por los y las aprendices. Algunos participantes, por ejemplo, afirmaban que su debilidad en el aprendizaje del inglés residía en la comprensión auditiva, tema común entre los aprendices de un segundo idioma.

La estrategia que se reportó con más frecuencia para el abordaje de este problema era escuchar música en inglés o ver películas o programas de televisión en ese idioma. Tales prácticas, sin embargo, no surten el efecto deseado, ya que no se trata únicamente de exponerse al idioma, sino también de poner en práctica técnicas de comprensión específicas dentro de ejercicios debidamente planificados.

Otro inconveniente relacionado con la autopercepción de alumnos y alumnas fue que, aunque seatribuía a la comprensión auditiva gran parte de la afectación en el desempeño académico, diferentes instrumentos de evaluación, así como la valoración cualitativa de los docentes evidenciaron deficiencias generalizadas en el uso del idioma y carencias para la producción escrita. En otras palabras, el plan de mejora debía enfocarse en el reforzamiento mismo de la estructura gramatical del idioma para lograr una comunicación eficiente en forma escrita u oral.

La investigación pudo determinar que los conocimientos adquiridos por los participantes sobre el aprendizaje en general realmente forman parte estable de los saberes acumulados a través de su experiencia de vida. Todos los alumnos fueron capaces de hablar ampliamente de sus creencias ya que las tienen a su disposición en su memoria, incluso de forma automática. Este conocimiento, sin embargo, es falible ya que puede originarse en creencias de uso generalizado más que en datos empíricos. El conflicto estriba en que el conocimiento personal de los estudiantes es el primer filtro para analizar objetivos de estudio y, sin duda, influye también en la selección de las estrategias que se emplean y la forma de autoevaluarse.

Pese a que los y las estudiantes involucrados en la investigación aprobaron las asignaturas de inglés de su primer cuatrimestre en la Carrera, se encontraron diferencias con respecto a sus calificaciones y sus competencias en el uso del idioma. Se hizo evidente que cada uno de los participantes había acumulado una serie de experiencias previas -positivas o negativasrelativas al aprendizaje en general y la adquisición del inglés en particular.

Esto concuerda con lo expuesto por Schraw (1994) quien encontró que los estudiantes adultos tienden a diferir en cuanto a la utilización de habilidades metacognitivas de regulación, aunque no en cuanto a su CMC. El aprendiz va modificando su $\mathrm{CMC}$, de manera consciente o inconsciente conforme avanza en su formación académica (Wenden, 1994). De tal manera, era esperable que los participantes del estudio que experimentaron mayor dificultad en su primer cuatrimestre podrían lograr un mejor desempeño a futuro si se les brinda entrenamiento para optimizar su CMC.

Por una parte, en relación con el conocimiento de la tarea (el estudio del inglés a distancia) algunos de los participantes precisaron su conocimiento; de igual manera, la dinámica y la naturaleza de la carrera a la que habían ingresado. Por otra parte, todos explicaron lo que para ellos eran características que definían al "buen aprendiz de inglés a distancia"; a saber: aptitud natural para el idioma, interés 
por la cultura de la lengua meta, competencia comunicativa, disposición para la lectura, compromiso, organización, disciplina, práctica constante, pasión por la carrera y conocimiento de sí mismos para identificar debilidades y autogestionar los apoyos necesarios. En general, los estudiantes evidenciaron comprensión de las demandas de su rol como estudiantes de un idioma a distancia.

El conocimiento de la tarea también se demostró en los aspectos que los participantes identificaron como habilidades necesarias para estudiar en la carrera de Enseñanza del Inglés: práctica constante, disciplina, compromiso y organización, entre otros. Se logró identificar la existencia de lo que Schraw y Moshman (1995) describen como teorías metacognitivas informales, marcos mentales que integran el CMC en forma explícita. No obstante, esto se realizó de manera fragmentaria, dado que en varios casos no se han comprendido aún las acciones que deben tomarse para lograr un aprendizaje eficaz (es decir, saber por qué debe hacerse algo) y este conocimiento tampoco se ha puesto a disposición para utilizarse a cabalidad.

Algunos estudiantes manejan teorías metacognitivas formales que se evidencian mediante nociones explícitas, estructuradas o integradas. Dichos aprendices son capaces de afirmar y clarificar los conocimientos que poseen sobre el proceso de aprendizaje y, por lo tanto, podrían aplicarlos de manera óptima en el futuro. Quienes cuentan con conocimientos generales sobre cómo aprender serán capaces de controlar y mejorar su propia instrucción (Schraw y Moshman, 1995).

En cuanto al conocimiento de la estrategia, los datos obtenidos mostraron que los estudiantes conocían diversas estrategias cognitivas, metacognitivas y de regulación de recursos. Todos ellos empleaban algunas de esas técnicas en sus estudios. Por ejemplo, se citó la memorización, una estrategia básica de ensayo que implica re- citar o nombrar objetos de una lista para aprender y es efectiva para realizar tareas simples y activar la información en la memoria de trabajo, no en la memoria a largo plazo (Pintrich, 2004). Otra estrategia que se citó fue la elaboración, la cual es muy útil para almacenar información en la memoria a largo plazo mediante la construcción de conexiones internas entre los elementos por aprender. Este tipo de estrategias incluyen el parafraseo, la elaboración de resúmenes, analogías y la toma de notas.

Se señalaron también las estrategias de planificación, como la asociación del conocimiento previo con la tarea de estudiar el inglés. Estas relaciones ayudan a activar aspectos relevantes de la experiencia previa para organizar y comprender el material de manera más fácil. Además, se reportó el uso de estrategias cognitivas de organización y gestión de recursos, las cuales ayudan a los alumnos y alumnas a seleccionar información adecuada y construir conexiones entre los contenidos que deben aprenderse, en un esfuerzo activo y consciente para mejorar el aprendizaje.

De manera adicional, se señaló el empleo de estrategias de compensación afectiva, pensamiento crítico y aplicación de conocimientos anteriores a situaciones nuevas para resolver problemas, tomar decisiones o hacer evaluaciones críticas con respecto a normas de excelencia. Los y las estudiantes en este estudio evidenciaron, en mayor o menor grado, conocimiento del automonitoreo o supervisión, y dieron seguimiento a las áreas en las cuales, a su criterio, presentan algún tipo de deficiencia.

Sobre el conocimiento contextual, los participantes explicaron cómo el estudio a distancia los había llevado a modificar hábitos de estudio y, sobre todo, la administración del tiempo. Todos describieron las ventajas que, en su opinión, esta modalidad de aprendizaje tiene en comparación con la modalidad tradicional o presencial. Por ejemplo, mencionaron la 
pertinencia y puntualidad de la instrucción y la flexibilidad encuantoal ritmoyestilodelaprendiz.

Por otra parte, reconocieron que esta modalidad, debido a sus particularidades y demandas, no es una opción para todas las personas. Indicaron que la asignación y ritmo de trabajo más allá de la tutoría son fuertes, en especial cuando se añaden a las responsabilidades familiares y laborales.

Se demostró clara consciencia de que el estudiante de la Carrera de Enseñanza del Inglés debe ejercer - de manera autónomacontrol sobre la información que necesita, sus tiempos ante las demandas de cada asignatura y la organización de actividades. Además, aseveraron los participantes, debe tomarse consciencia de las necesidades y características personales para adecuar su forma de estudiar y hacer un manejo eficiente del tiempo y de los recursos.

\section{Conclusiones}

El estudio de caso múltiple realizado en la Carrera de Enseñanza del inglés muestra que los estudiantes a distancia se enfrentan a menudo con nuevas tareas que requieren conocimientos y habilidades que aún no han adquirido. Por consiguiente, necesitan conocer estrategias generales para el estudio a distancia y el pensamiento crítico, a fin de probar su efectividad en labores nuevas o desafiantes.

Los hallazgos de investigación apuntan a la necesidad de implementar una instrucción metacognitiva. No obstante, es fundamental acotar que el entrenamiento metacognitivo no sustituye el aprendizaje de contenidos particulares de una disciplina, más bien, es un complemento del proceso integral de formación. En tal sentido, toda estrategia que se adopte para el desarrollo metacognitivo debe integrarse en el proceso general de desarrollo de destrezas en la lengua meta.
Un punto aparte en los resultados de la investigación lo constituye la dimensión denominada "conocimiento de la persona". Se comprobó que dicha área es crucial para el estudio a distancia ya que puede ser tanto un estímulo como una limitación.

Los estudiantes que conocen sus propias fortalezas y debilidades ajustan su cognición y pensamiento para ser más adaptables a diversas tareas y, así, facilitar la adquisición de nuevos contenidos. Los que poseen un conocimiento incompleto serán menos capaces de adaptarse a diferentes situaciones y regular su propio aprendizaje. Como se observó durante el estudio, las creencias personales constituyen un poderoso filtro de la realidad.

Existen varias implicaciones de las relaciones entre el CMC, el aprendizaje, la enseñanza y la evaluación. En cuanto a la instrucción, es vital enseñar de manera explícita el conocimiento metacognitivo. Los docentes pueden hacerlo en las lecciones o en unidades específicas, aunque resulta más efectivo que el CMC se incorpore en las lecciones regulares basadas en el contenido habitual de las materias.

Las estrategias generales de pensamiento y solución de problemas, por ejemplo, pueden enseñarse en el contexto del estudio del inglés y el aprendizaje sería más eficaz si se vinculara al contenido específico de la gramática y la fonética, no en abstracto. En algunas áreas, tales como la lectura o la escritura, la enseñanza del $\mathrm{CMC}$ con respecto a ciertas estrategias generales de comprensión sería pertinente y deseable.

Los docentes pueden incluir objetivos para la enseñanza de conocimientos metacognitivos en la unidad regular, así como en los contenidos específicos de la asignatura y, luego, enseñar y evaluar usando el CMC. Por ejemplo, puede emplear un breve cuestionario como el siguiente para alguna tarea de aprendizaje activo: 
o Plantee tres preguntas acerca de los conceptos explorados en esta tarea que usted todavía no puede responder.

o Describa al menos dos ideas relacionadas con esta tarea que le parecen confusas.

o ¿Está de acuerdo con la frase "he aprendido mucho" en esta tarea? ¿Por qué? ¿Por qué no?

o Piense en la forma en la que abordó esta tarea. Compare su estrategia con la que utilizó para completar la última asignación de esta materia. ¿Qué consejo se daría a usted mismo basándose en lo que ahora sabe, si estuviera empezando la asignación?

Durante la lección, el tutor puede identificar ocasiones en que el conocimiento metacognitivo se puede discutir. El lenguaje relativo a la cognición compartido entre pares, así como entre tutores y alumnos, ayuda a los estudiantes a ser más conscientes de su propio CMC. Mientras los aprendices escuchan o ven cómo sus compañeros de grupo abordan una determinada tarea, pueden comparar sus propias estrategias y valorarlas. Este tipo de discurso y debate ayuda a que la cognición y el aprendizaje sean menos opacos para los estudiantes y no se perciban como algo que sucede de manera misteriosa.

Otra forma de enseñanza efectiva del CMC es la demostración de estrategias, acompañada dela explicación correspondiente.Porejemplo,cuando la tutora o el tutor está explicando un ejercicio a la clase, puede hablar en voz alta sobre sus propios procesos cognitivos a medida que resuelve el problema. Este método proporciona un modelo para los estudiantes, mostrándoles cómo aplicar estrategias para resolver problemas reales.

Además, el docente también podría exponer las razones por las que está empleando esa técnica en particular para resolver un ejercicio específico, de manera que se propicie la discusión con los alumnos sobre cuestiones relacionadas con el conocimiento condicional que gobierna el cuándo y el por qué usar diferentes procedimientos.

Si los docentes consideran el conocimiento metacognitivo como parte de su dinámica del aula, entonces también deben poner atención a lo que los estudiantes expresen sobre su propia cognición. Con base en lo anterior, los tutores podrán comprender el nivel general y la profundidad del CMC de sus alumnos. En muchos aspectos, esto no es diferente de los diagnósticos que efectúan los docentes de idioma al inicio de un curso para evaluar el nivel de conocimientos de sus alumnos.

Se recomienda que los docentes se comuniquen con sus alumnos de forma individual o en grupos pequeños para estimar sus niveles de CMC. Pueden aplicar procedimientos como la entrevista y los cuestionarios más formales para evaluar la conciencia de los discentes acerca de sus estrategias de aprendizaje, así como sus conocimientos sobre diferentes tareas y sus contextos (Pintrich et al., 2000).

Se ha insistido en el hecho de que el conocimiento de la persona constituye una dimensión determinante del CMC (Flavell, 1979), razón por la cual la evaluación debe propiciar que los estudiantes tengan la oportunidad de medir sus propias fortalezas y debilidades. Aunque esto no siempre se podrá hacer cuando 
se manejen grupos grandes, es necesario - por razones motivacionales - una autoevaluación más privada, entre el tutor y el alumno (Pintrich y Schunk, 2002).

Mediante la comunicación en línea, los estudiantes están en capacidad de reunirse en forma individual con sus tutores para discutir las percepciones de sus fortalezas y debilidades; los docentes, a su vez, pueden proporcionarles realimentación sobre estas percepciones. A manera de ilustración, la evaluación mediante portafoliosofrecealosestudiantesla oportunidad de reflexionar sobre su trabajo y acrecentar su autoconocimiento.

Los tutores y tutoras también deben ayudar a los aprendices a desarrollar un enfoque más reflexivo y autodirigido en la adquisición de la L2. La mayor parte de los docentes de idioma tienen por objetivo proporcionar a sus estudiantes la instrucción que facilita el incremento de la autonomía lingüística. Sin embargo, esta investigación sugiere que los alumnos también necesitan orientación para mejorar y ampliar sus conocimientos sobre el aprendizaje en sí mismo para ser más autónomos en su enfoque hacia el perfeccionamiento de la lengua que deberán enseñar en un futuro próximo.

\section{Referencias}

Cohen, A. D. (2011). Second Language Learner Strategies. En Hinkel, E. (Ed.) Handbook of Research in Second Language Teaching and Learning, 2 (pp. 681-698). New York, NY: Routledge.

Creswell, J. W. (2017). Qualitative Inquiry and Research Design: Choosing Among Five Approaches. New York. Sage.

Dewitz, P., \& Graves, M. F. (2014). Teaching for transfer in the common core era. The Reading Teacher, 68(2), 149-158. https://doi. org/10.1002/trtr.1290.
Duff, P. (2012). How to Carry out Case Study Research. En A. Mackey \& S. M. Gass (Eds.), Research Methods in Second Language Acquisition: A Practical Guide (pp. 95-116). New York, NY: Wiley-Blackwell.

Duff, P. (2008). Case Study Research in Applied Linguistics. New York, NY: Erlbaum/Taylor \& Francis.

Flavell, J. H. (1979). Metacognition and Cognitive Monitoring: A New Area of Cognitive-Developmental Inquiry. American Psychologist, 34(10), 906-911. DOI: http://dx.doi. org/10.1037/0003-066X.34.10.906

Flavell, J. H. (1987). Speculation about the $\mathrm{Na}$ ture and Development of Metacognition. Metacognition, Motivation and Understanding. Hillsdale, NJ: Lawrence Erlbaum.

Gay, L. R., Mills, G. E. y Airasian, P. W. (2013). Educational Research: Pearson New International Edition: Competencies for Analysis and Applications. Pearson Higher Ed.

Graham, S. (2004). Giving up on Modern Foreign Languages? Students' Perceptions of Learning French. Modern Language Journal, 88(2), 171-191.

Gurbin, T. (2015). Metacognition and Technology Adoption: Exploring Influences. Procedia-Social and Behavioral Sciences, 191, 15761582.

Hampel, R. y de los Arcos, B. (2013). Interacting at a Distance: A Critical Review of the Role of ICT in Developing the LearnerContext Interface in a University Language Programme. Innovation in Language Learning and Teaching 7(2), 158-178.

Hernández, R., Baptista, P. y Fernández, C. (2010). Metodología de la Investigación (5ta. $E d)$. D.F., México: McGraw-Hill.

Hong, S. y Jung, I. (2011). The distance learner competencies: A three-phased empirical 
approach. Education Technology Research and Development, 59, 21-42.

Hung, M.-L., Chou, C., Chen, C.-H. \& Own, Z. (2010). Learner readiness for online learning: Scale development and student perceptions. Computers $\mathcal{E}$ Education, 55(3), 1080-1090.

Kempe, V. (2011). Individual differences in adult second language learning: A cognitive perspective. Scottish Languages Review, $23,15-22$.

Kormos, J. y Csizér, K. (2014). The Interaction of Motivation, Self-Regulatory Strategies, and Autonomous Learning Behavior in Different Learner Groups. TESOL Quarterly 48(2), 275-299.

Pintrich, P. R. (2004). A conceptual framework for assessing motivation and self-regulated learning in college students. Educational psychology review, 16(4), 385-407.

Pintrich, P. R., \& Schunk, D. H. (2002). Motivation in education: Theory. Research, and Applications, (2nd Ed.). Merrill Prentice Hall, Columbus, Ohio.

Raoofi, S., Chan, S. H., Mukundan, J. y Rashid, S. M. (2013). Metacognition and Second/Foreign Language Learning. English Language Teaching 7(1), 36.
Richards, J. C., \& Rodgers, T. S. (2014). Approaches and methods in language teaching. Cambridge University Press.

Schraw, G., \& Dennison, R. S. (1994). Assessing metacognitive awareness. Contemporary educational psychology, 19(4), 460-475.

Schraw, G., \& Moshman, D. (1995). Metacognitive theories. Educational Psychology Review 7(4), 351-371.

Stake, R. E. (2010). Qualitative research: Studying how things work. Guilford Press.

Wenden, A. L. (1994). Learner representations in language learning: relevance and function. En: Proceedings of the international conference "AUTONOMY 2000:" the development of learning independence in language learning, pp. 234-253.

White, C. (2014). The distance learning of foreign languages: A research agenda. Language Teaching, 47(04), 538-553.

White, C. (1999). The Metacognitive Knowledge of Distance Learners. Open Learning: The Journal of Open, Distance and e Learning, 14(3), 37-46. 